\title{
Discrimination of 3-D shape and 3-D curvature from motion in active vision
}

\author{
WIM J. M. VAN DAMME, FERRY H. OOSTERHOFF, and WIM A. VAN DE GRIND \\ Utrecht University, Utrecht, The Netherlands
}

\begin{abstract}
We examined the ability of human observers to discriminate between different 3-D quadratic surfaces defined by motion, and with head position fed back to the stimulus to provide an up-todate dynamical perspective view. We tested whether 3-D shape or 3-D curvature would affect discrimination performance. It appeared that discrimination of 3-D quadratic shape clearly depended on shape but not on the amount of curvature. Even when the amount of curvature was randomized, subjects' performance was not altered. On the other hand, the discrimination of 3-D curvature clearly depended linearly on curvature with Weber fractions of $20 \%$ on the average and, to a small degree, on 3-D shape. The experiment shows that observers can easily separate 3-D shape and 3-D curvature, and that Koenderink's shape index and curvedness provide a convenient way to specify shape. These results warn us against using just any arbitrary 3-D shape in 3-D shape perception tasks and indicate, for example, that emphasizing 3-D shape in computer displays by exaggerating curvature does not have any effect.
\end{abstract}

Since Wallach and O'Connell (1953) demonstrated that three-dimensional (3-D) shape could be extracted from orthographic projections of rotating objects, there have been numerous studies of this phenomenon, which is commonly known as the kinetic depth effect. Such psychophysical studies include, for example, those on object rigidity (Todd, 1984), perceived depth or curvature (Dosher, Landy, \& Sperling, 1989; Norman \& Lappin, 1992; Saidpour, Braunstein, \& Hoffman, 1992), minimal conditions for the perception of 3-D shape from motion in multidot displays (Sperling, Landy, Dosher, \& Perkins, 1989; Todd \& Norman, 1991; Treue, Husain, \& Andersen, 1991), projection methods (Todd, 1984), and token dependency (Landy, Dosher, Sperling, \& Perkins, 1991).

The most common way to study the phenomenon of 3-D shape from motion is to use random dot cinematograms of moving 3-D objects. The objects under study are mostly cylinders, planes, or spheres. There is no reason to assume that the results of these studies hold for all kinds of 3-D shapes, but most studies have not included a wide range of shapes or curvature values. On the other hand, the importance of a multiobject study has been pointed out by several authors. Norman and Lappin (1992) reported differences in discriminability between cylinders and spheres, spheres and planes, and cylinders and planes. Sperling et al. (1989) used a finite set of 53 different 3-D shapes in a shape identification task, but did not report quantitative differences in performance for different 3-D shapes. Braunstein and Andersen (1984) noted that spheres,

This research was sponsored by the SPIN project "3-D Computer Vision" of the Dutch Ministry of Economic Affairs. Correspondence should be addressed to W. J. M. van Damme, Department of Comparative Physiology, Utrecht University, Padualaan 8, $3584 \mathrm{CH}$ Utrecht, The Netherlands (e-mail: wim@neuretp.biol.ruu.nl). for example, might have certain heuristic topological characteristics that would make them special objects, not suitable for a generalization to other 3-D objects. It has to be examined yet what exactly these "heuristic" properties are. It is well possible that a certain systematic 3-D shape dependency in structure from motion is responsible for this effect. A quantitative examination of 3-D shape perception obviously requires a mathematical definition of 3-D shape. On the other hand, if we want to study 3-D shape perception in psychophysical experiments, this definition must be in some way ecologically valid so that human observers can handle it in an intuitive way.

In previous research (van Damme \& van de Grind, 1993), we presented such a mathematical definition of 3-D shape and showed in a psychophysical experiment that it is close to an intuitive idea of 3-D shape. We showed that identification of 3-D shape did not depend on the amount of 3-D curvature, and that identification performance of human observers depended on the 3-D shape. The experiment, however, did not provide precise quantitative information on discriminability or sensitivity to 3-D shape and 3-D curvature. The present study is meant to provide that information.

The goal of the present study was twofold. First, the effect of 3-D shape and 3-D curvature magnitude on the discrimination performance of human observers with respect to different 3-D quadratic shapes was examined. Second, the effect of the same parameters on discriminability of 3-D curvature was examined. Together, the results of these investigations quantify the dependency on 3-D shape and 3-D curvature in the structure from motion process. A continuous range of 3-D quadratic shapes was used, including the cylinder- and sphere-like shapes often used in other studies. Increment thresholds in shape discrimination (Experiment 1) and in 3-D curvature dis- 


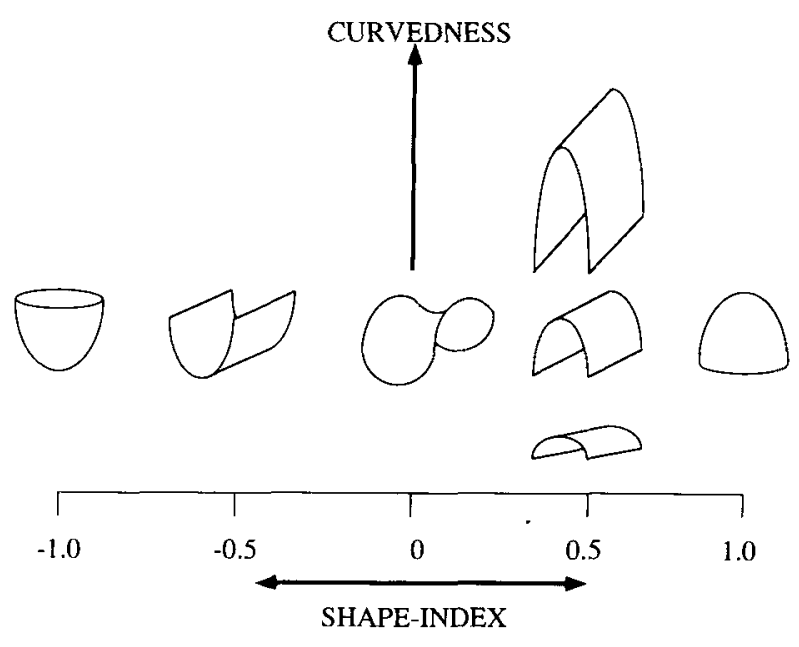

Figure 1. The shape index $S$ and curvedness $C$ as two parameters describing 3-D quadratic shape. $S$ ranges from -1 to +1 ; this figure shows some specific quadratic shapes as an example: $S=-1$, concave umbilic shape; $S=+1$, convex umbilic shape; $S=-0.5$, concave cylinder shape; $S=+0.5$, convex cylinder; $S=0$, symmetric saddle. $C$ ranges from 0 to infinity, where $C=0$ stands for an undetermined shape. Increasing the curvedness does not change the shape of the surface.

crimination (Experiment 2) were determined. The shape discrimination was measured for multiple values of 3-D curvature, whereas 3-D curvature discrimination was measured for multiple 3-D shapes. Whenever the term 3-D curvature is used in this paper, it refers to curvature in depth, which is the curvature of a space curve that lies on a surface patch. Because the term curvature is sometimes used both to denote 2-D curvature (curvature in the frontoparallel plane) and 3-D curvature (curvature of a space curve), which is somewhat confusing, it is important to emphasize that in this paper 2-D curvature and 3-D curvature are different things.

The motion that is responsible for the 3-D sensation can be induced by movement of the object or by movement of the observer. There are indications that proprioceptive information, such as that from the vestibular system or from muscular activity, influences the interpretation of retinal signals (Roll, Velay, \& Roll 1991). Therefore, it seems reasonable to assume that " 3 -D shape from active vision" is different from "3-D shape from moving objects." Active "vision" is nowadays extensively used in machine vision and robotics-artificial visual systems using an often primitive form of camera motion to obtain depth information about the world. Active vision is a familiar method of obtaining depth information throughout the animal kingdom, and many studies on peering mechanisms have been published in the neurobiological literature.

In this paper, only the active exploration of 3-D objects by human observers is considered. No thorough comparison is made between active and passive vision, nor do we provide an extensive analysis of all conceivable forms of active vision. Our primary goal was to examine the perception of 3-D shape and 3-D curvature by using linear frontoparallel (left-right) exploratory head movements. Such experiments were introduced by Rogers and Graham $(1979,1983)$.

Clearly, it is impossible to describe the infinite set of all possible 3-D shapes by resorting to a single parameter. In this paper, a subset of quadratic shapes is chosen, which are defined by the formula: $Z=0.5 *\left(K_{\min } *\right.$ $\left.X^{2}+K_{\max } * Y^{2}\right)$, where $K_{\min }$ and $K_{\max }$ are the principal curvatures (Coxeter, 1961).

To quantify and qualify 3-D quadratic shape in a more systematic way, two parameters will be used: a shape index, $S$, and a curvedness, $C$, (Koenderink, 1990). In previous studies (de Vries, Kappers, \& Koenderink, 1993; van Damme \& van de Grind, 1993), it has been shown that these parameters are very suitable to quantify local 3-D quadratic shape, both theoretically and in psychophysical experiments. Strictly speaking, the term local shape implies that the shape index describes the shape only in one point. However, it is possible to speak of the shape index and the curvedness of a surface patch when actually the shape index and the curvedness in the point that is surrounded by the patch are meant. The formal definition of shape index and curvedness can be given as follows (Koenderink, 1990):

$$
\begin{gathered}
S=-\frac{2}{\pi} \arctan \frac{K_{\max }+K_{\min }}{K_{\max }-K_{\min }} \\
C=\sqrt{\frac{K_{\min }^{2}+K_{\max }^{2}}{2}} .
\end{gathered}
$$

Figure 1 shows the shape index scale together with some examples of familiar quadratic surface patches at the appropriate positions along this scale; Figure 2 shows some more examples of less familiar quadratic shapes with the corresponding shape index and curvedness values. The units are in meters. Following this definition, the curvedness in a generic point covers the 3-D curvature in that point of all space curves passing through that point. This means that if the curvedness of a surface patch is known, the curvature of all space curves in that point is known.

\section{METHOD}

Stimuli

Chinrest-position-dependent (dynamic) random dot patterns were generated on a computer screen. One image was made as follows: First, random $x$ and $y$ coordinates were generated within a square patch of $0.4 \times 0.4 \mathrm{~m}$. Next, the corresponding $z$ value was computed with the formula $Z=0.5 *\left(K_{\min } * X^{2}+K_{\max } * Y^{2}\right)$. In the experimental setup, $z$ is the coordinate along the line of sight (with $z=0$ being the plane of projection), where $x$ and $y$ represent orthogonal coordinates in the frontoparallel plane. The minus sign serves to make convex curvatures point toward the observer and concave curvatures point away from the observer, being in close agreement with the intuitive notion of "convex" and "concave." The points in 3-D space were then rotated around the line of sight through a random angle between $0^{\circ}$ and $180^{\circ}$. Since all quadratic surfaces have two lines of symmetry, this rotation covers the full orientation spectrum of a quadratic surface. The random orientation 

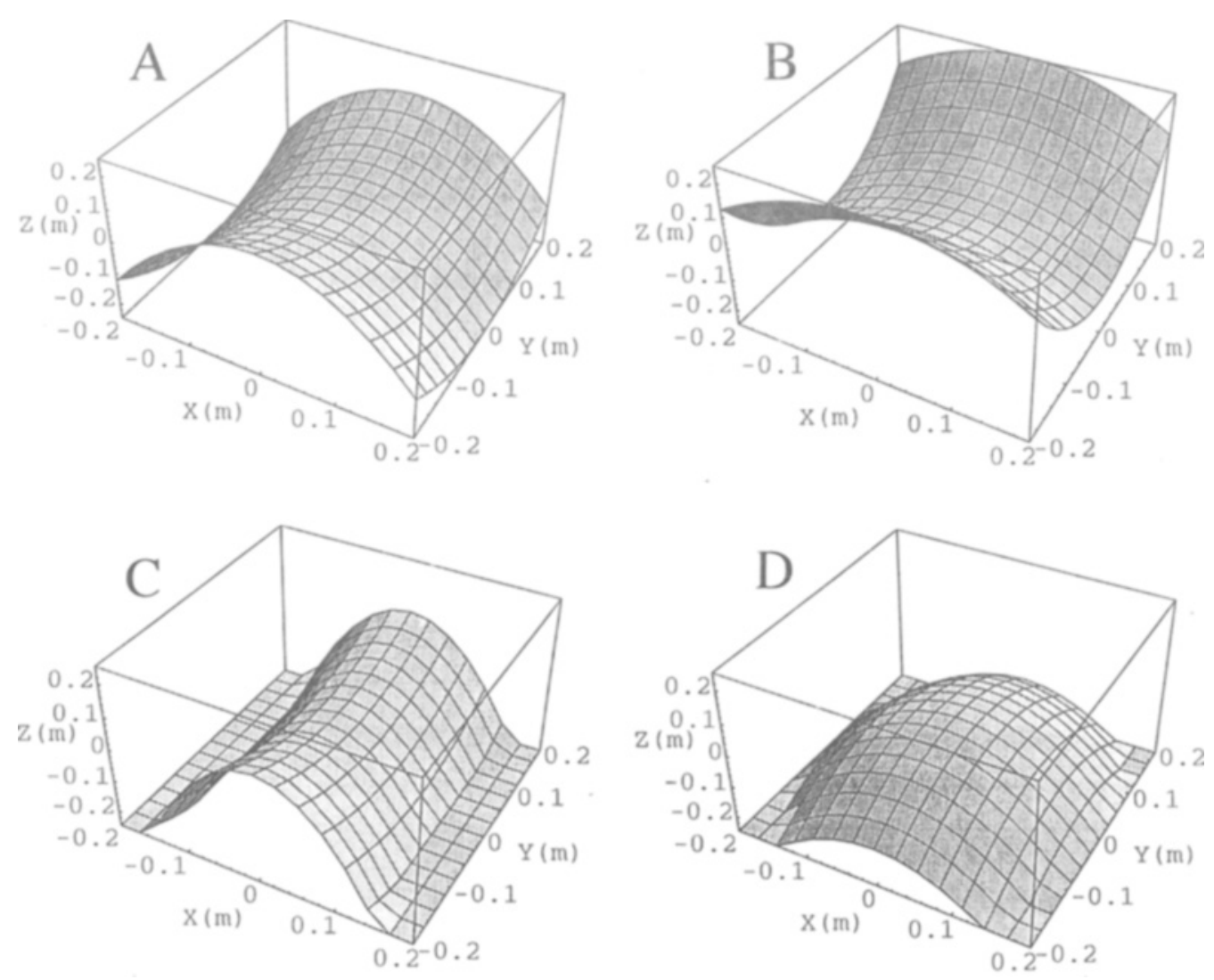

Figure 2. Some examples of quadratic shapes: (A) $C=10 / \mathrm{m}, S=0.2$. (B) $C=10 / \mathrm{m}, S=-0.2$. (C) $C=20 / \mathrm{m}$, $S=0.4$. (D) $C=10 / \mathrm{m}, S=0.8$.

avoids possible orientation anisotropies such as those reported by Droulez and Cornilleau-Pérès (1990) and Norman and Lappin (1992).

A perspective projection was made onto the plane of the computer screen, using the actual chin position that was measured by the movable chinrest. To avoid density changes' having too much impact, the overall density was kept below $1 \%$ by imposing a limit on the total number of random dots. The dot density was uniform only when the stimulus was viewed in frontoparallel position, but in other positions there was a slight increase/decrease in density that could (at least in principle) act as an artifactual cue. (For an extensive discussion of the effect of density, see, e.g., Sperling, Landy, \& Dosher, 1990.) Removing the density cue introduces scintillation as a possible cue. To eliminate both density changes and scintillation is principally impossible. During presentation of the stimulus, a square mask occluded the deforming borders of the patch, which otherwise would have been a cue for the shape of the surface. The stimuli consisted of 1,500 dots, the viewing distance was $2.36 \mathrm{~m}$, and the stimulus size on the computer screen was $0.27 \times 0.27 \mathrm{~m}$, so the viewing angle was $6.5^{\circ} \times 6.5^{\circ}$. Because the mask that was applied obscures part of the dots, the number of actually visible dots was less than 1,500 (on the average, about 800 ). This number varies slightly, of course, when the stimulus moves, since dots disappear at one side and appear at the other side.

Information about the shape of the surface was present only when the observer's head was moved, inducing movement of the dots on the screen. When the dots were not moving no information about the shape was visible; it was just a static random dot display.

Eye movements were not restricted during the presentation of the stimulus. Each stimulus was visible for $3 \mathrm{sec}$. There was no self-occlusion of the surface.

\section{Apparatus}

The chinrest could be moved along a rail parallel to the screen over a distance of $0.397 \mathrm{~m}$ (Figure 3).

A Trinitron GDM 1950/1952 19-in. RGB computer monitor was used, with a frame rate of $60.0 \mathrm{~Hz}$ and a pixel size of $0.35 \mathrm{~mm}$. The dots were drawn within two frame durations of the computer monitor, so the actual refresh rate of the stimulus was $30.0 \mathrm{~Hz}$. The chinrest was mechanically coupled to a potentiometer, which rotated as the chinrest was moved. The voltage across the potentiometer was measured by an analog/digital interface card (National Instruments NB-MIO 16L) at $100 \mathrm{~K}$ samples/sec. From this voltage the position of the chin was calculated by a Macintosh IIfx computer. The chinrest system was calibrated before each experimen-

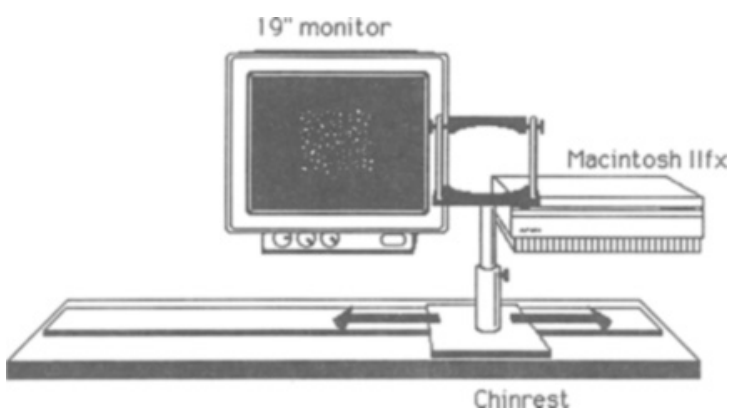

Figure 3. The experimental setup. 

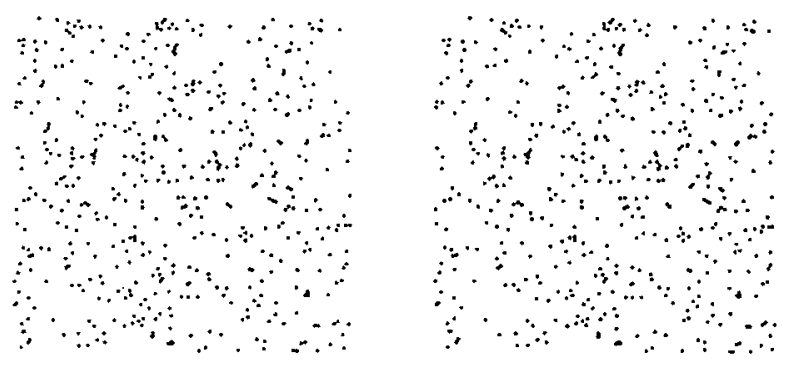

Figure 4. Two example stimuli. The stimuli were actually $0.27 \times$ $0.27 \mathrm{~m}$ and viewed from $2.36 \mathrm{~m}$. These are the random dot patterns corresponding to a chinrest position in the middle and $6 \mathrm{~cm}$ to the right. The figure can be viewed stereoscopically. It represents a quadratic shape with shape index $S=-0.3$ and curvedness $C=10 / \mathrm{m}$.

tal session. Chin positions were measured with a resolution of $0.255 \mathrm{~mm}$. The lag time of the system (i.e., the time between movement of the chinrest and detection of the movement) was mainly determined by the mechanical hysteresis of the chinrest and the sampling-rate of the interface board, and was typically $0.01 \mathrm{msec}$, which was well under the video refresh rate $(16 \mathrm{msec})$. Given the chin position, the corresponding eye position was calculated. For convenience, the position of the eye was considered to be $3 \mathrm{~cm}$ to the left or to the right from the chin position, depending on which eye was used for observation.

Figure 4 shows the stimulus at two different chinrest positions: a view from the middle chinrest position and a chinrest position that is $6 \mathrm{~cm}$ toward the right. This figure can therefore be viewed stereoscopically. It represents a quadratic shape with $S=+0.3$ and $C=10 / \mathrm{m}$.

\section{Procedure}

Experiment 1: Discrimination of 3-D quadratic shape. To obtain just noticeable differences (JNDs) between quadratic surfaces, a two-alternative forced choice (2AFC) procedure was used. Subjects were shown series of 40 pairs of quadratic surfaces, with each surface represented by its random dot version. All observations were performed monocularly. During a given series, one of the pair of images always had the same shape index. This image will henceforth be called the reference image. At the beginning of a new series, the subjects were informed about the value of this reference shape index. The shape index of the other image in the pair of images (henceforth called the test image) was unknown to the subjects. The subjects were also informed about the shape index of the first test image in the staircase. The task of the subjects was to indicate which one of the two images in each presented pair was the reference image. The shape index of the test image was varied according to a staircase procedure; that is to say, if a subject made a correct response, the difference in shape index between test image and reference image was decreased, and if a subject made a false response, the difference in shape index between test image and reference image was increased. The staircase was designed in such a way that subjects could get closer to the reference image or further from the reference image, but could never "pass" the reference image. In this way, a distinction can be made between approaching the reference image from above (test shape index $>$ reference shape index) and approaching the reference image from below (test shape index < reference shape index). The JND when approaching from below might be different from the JND when approaching from above. To correct for this possible asymmetry, each reference shape index was approached twice from below and twice from above. The four obtained JNDs were then averaged.
The amounts of increase and decrease in a staircase were chosen in such a way that an ideal response would lead to a $75 \%$ correct score.

The first 10 responses within a series of $\mathbf{4 0}$ were used to arrive at threshold level and were discarded in further analysis. The remaining 30 responses were averaged. The resulting mean value was then combined with those from the three other trials on the same reference shape index to determine the average JND for this shape index.

A pilot experiment showed that most JNDs were below 0.2 on the shape index scale. Therefore, all staircase procedures started with $S_{\text {test }}=S$ ref \pm 0.2 . To obtain JNDs for the whole range of shape indices, the above-mentioned staircase procedure was repeated for 11 different shape index values $(-1.0,-0.8,-0.6,-0.4,-0.2$, $0.0,0.2,0.4,0.6,0.8$, and 1.0). Note that some of these reference shape indices correspond to shapes that might have certain "special" geometric characterictics. For example, $S=0.0$ is a perfect symmetrical saddle shape, and $S= \pm 1.0$ corresponds to perfect symmetrical umbilics. There are more such possible "special" shapes ( $S= \pm 0.5$, perfect parabolic shapes), but not all are used as reference shape.

The values $S=-1.0$ and $S=+1.0$ pose some difficulties, since the first cannot be approached from below, and the second not from above. Therefore, those two reference shape indices were approached four times from above $(S=-1.0)$ and four times from below $(S=+1.0)$, respectively.

A quadratic surface is fully defined by the shape index and the curvedness. Within one series of 11 different reference shape indices, the curvedness was kept constant. To examine whether curvedness plays an important role in this task, the complete procedure was performed at two different values of curvedness: $C=$ $10 / \mathrm{m}$ and $C=20 / \mathrm{m}$. For the same reason, a random curvedness condition was created as a separate experimental condition. Should subjects use the curvedness as a cue, then the random curvedness should eliminate this possibility. In the random curvedness case, the curvedness could have a random value between $5 / \mathrm{m}$ and $20 / \mathrm{m}$.

In summary, for three different curvedness values, the JND values for 11 different shape indices were measured; each of them was measured four times as an average of a series of 30 pairs of images.

Experiment 2: Discrimination of 3-D curvature. The procedure for this experiment resembled the procedure of Experiment 1, but now the roles of shape index and curvedness were reversed: In one staircase, the shape index was kept fixed $(1.0,-0.5,0.0$, 0.5 , or 1.0) and there were 40 pairs of images, one test image with curvedness $C_{\text {test }}$, and one reference image with curvedness $C_{\text {ref. }}$. In this experiment, $C_{\text {test }}>C_{\text {ref }}$, so the task for the subjects was now reformulated as, "Which one of the pair of images appeared as being the flattest?" Within one session, there were four values of curvedness: $1 / \mathrm{m}, 5 / \mathrm{m}, 10 / \mathrm{m}$, and $20 / \mathrm{m}$. Each curvedness was repeated four times. There was no random shape index condition in this experiment.

There is one main difference in stimulus characterization between this experiment and the previous one. Because curvedness discrimination can theoretically be performed by comparing the mean element speeds in the test and reference images, the average element speed was randomized. The effect of randomizing the mean speed is that the origin of the simulated surface now appeared as floating either slightly in front of or slightly behind the screen instead of being fixed to the screen. The randomization process was implemented by choosing the simulated distance between the origin of the surface and the observer defined as $Z=2.36 \mathrm{~m}+$ random $[-12.5 \mathrm{~cm},+12.5 \mathrm{~cm}]$.

\section{Subjects}

Three male subjects took part in Experiment 1-one of the authors (W.D.), and two doctoral students. In Experiment 2, there were 2 male subjects and 1 female subject, one of whom (W.D.) had participated in Experiment 1 as well, and the other of whom were 
undergraduate students. All subjects, except W.D., were naive with regard to the purpose of the experiment. All subjects had normal or corrected-to-normal vision. Each naive subject was trained in using the shape index, so that none had any problems in knowing by heart which 3-D quadratic shape corresponded to which shape index, and vice versa.

\section{RESULTS}

\section{General}

All subjects reported that they had a clear impression of a 3-D shape that was static and rigid. Spontaneous depth reversals were reported in some cases. The scores of individual staircases were well above chance.

\section{Experiment 1}

Figure 5 shows the results for the 3 subjects F.V., W.D., and I.H. In these figures, each data point depicts the just noticeable difference obtained at that particular reference shape index. The reference shape index is on the horizontal axis. Each figure contains the JNDs for the three different conditions $C=10 / \mathrm{m}, C=20 / \mathrm{m}$, and $C=$ random (between $5 / \mathrm{m}$ and $20 / \mathrm{m}$ ).

It is clear from Figure 5 that JNDs are not the same for each shape index. For 2 of the 3 subjects (W.D. and I.H.), there is a clear increase in JND around $S=0$ and near the outer parts of the $S$ scale $(S=-1.0$ and $S=$ 1.0). The lowest JNDs were measured for $S=-0.4$ and $C=10 / \mathrm{m}(0.0257$, W.D. $)$ and $S=0.4$ and $C=20 / \mathrm{m}$ $(0.0183$, I.H.). This means that Subjects I.H. and W.D. could tell the difference between two cylinder-like shapes best, and that they had more difficulties when discriminating two symmetrical hyperbolic shapes or two symmetrical elliptic shapes. For Subject F.V., there is no clear preference for either shape.

The results of this experiment can also be presented in terms of absolute depth differences. The largest depth differences between two patches occurred at the boundary, since we designed the stimuli in such a way that the center part of each stimulus always had the same depth. Therefore, the difference in depth was zero in the center, and had a maximum at the boundary. If the difference at the boundary is defined as $\Delta d$, then the smallest $\Delta d$ of all shapes that Subject W.D. was able to detect correctly in $75 \%$ of the cases was $2.345 \mathrm{~cm}$ for $S=-0.4$, which is less than $1 \%$ of the viewing distance $(2.36 \mathrm{~m})$. The 2 other subjects have similar values. Of course, this does not mean that subjects actually used this $\Delta d$ for making the decision. In fact, none of the subjects reported that they used depth differences at a certain point of the stimuli to make the decision.

A two-way (shape index $\times$ curvedness) analysis of variance (ANOVA) split by subject was performed on the data of Figure 5. The main effect of shape index was significant for all 3 subjects [F.V., $F(10,99)=10.722, p<$ .0001 ; I.H., $F(10,99)=8.500, p<.0001$; W.D., $F(10,99)=2.320, p=.017]$. The main effect of curved-
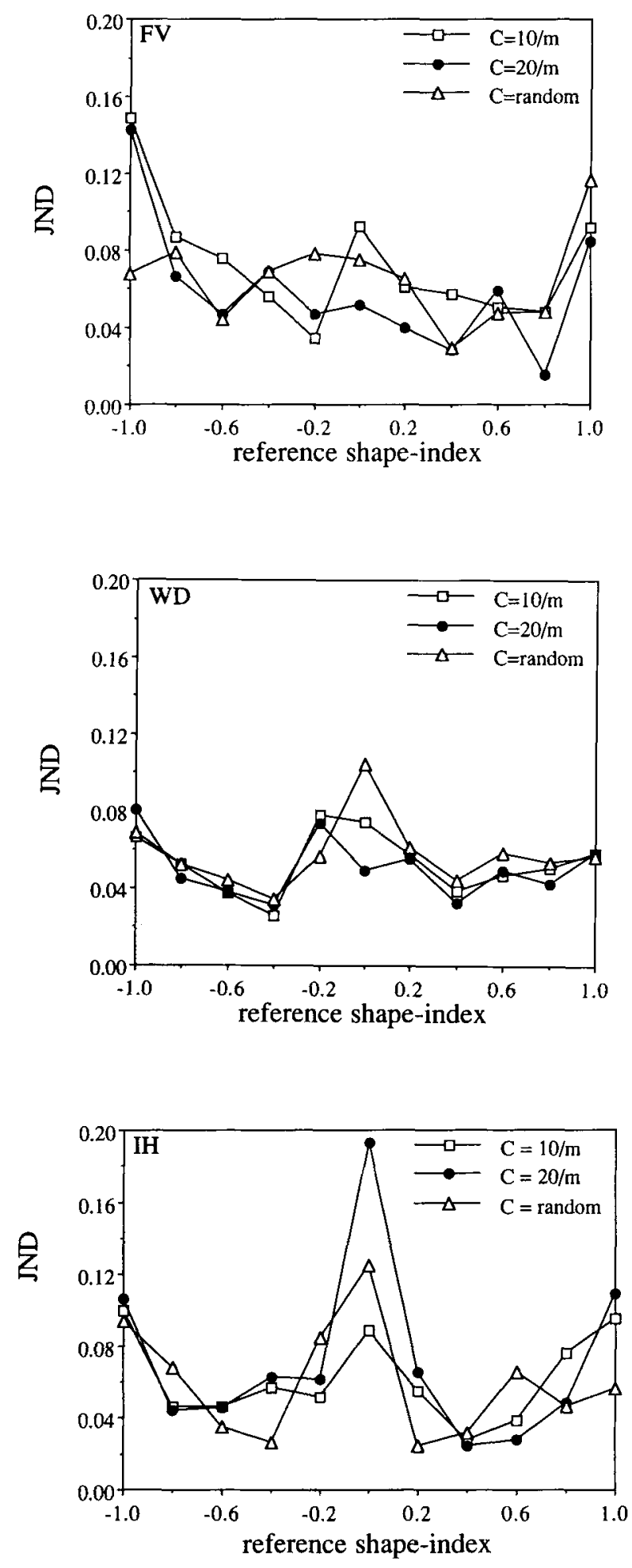

Figure 5. Just noticeable shape index differences for the 3 subjects F.V., W.D., and I.H. for the three conditions: squares, $C=$ $10 / \mathrm{m}$; filled circles, $C=20 / \mathrm{m}$; triangles, $C=$ random. Error bars denote standard errors. 
ness was not significant for either subject. There was only one case of a significant interaction between curvedness and shape index [F.V., $F(20,99)=2.328, p=.003]$.

From Figure 5 it might be concluded that only the specific values $S=1.0,0.0$, and -1.0 pop out with high JNDs, whereas the rest of the JNDs have more or less the same low value. This might be partly due to the finite shape index scale: because the end points at $S=1.0$ and $S=-1.0$ only have neighboring shapes in one direction, a different JND is measured at those points. However, an ANOVA on the data with the exclusion of these three cases $(S=1.0,0.0$, and -1.0$)$ still indicated a significant main effect of shape index for Subjects F.V. and I.H., although it is not so strong [F.V., $F(2,72)=4.189, p=$ .0006 ; I.H., $F(2,72)=2.252, p=.040$ ]. The main effect of curvedness was still not significant, and there were no significant interactions in this case.

\section{Experiment 2}

Figure 6 shows the results of the curvedness discrimination experiment for the 3 subjects W.D., K.Z., and S.M. Each panel shows the increment thresholds of curvedness at five different shape index values. Error bars are standard errors of the mean over the four repeated measurements.

Figure 6 shows that the thresholds increase (some almost linearly) with increasing curvedness, except at the lowest value, $C=1 / \mathrm{m}$. Figure 7 shows the same data as does Figure 6, but now plotted as a Weber fraction $\Delta C / C$. These figures demonstrate even more clearly that the lowest value of $C=1 / \mathrm{m}$ deviates strongly, but that the other curvedness values obey Weber's law reasonably well.

One reason for the strong deviation of the point $C=$ $1 / \mathrm{m}$ might be that the stimulus does not contain enough information to specify a smoothly curved surface. Just as in the case of random dot stereograms, a smoothly curved surface is deformed into a stepwise shaped "pyramid" because of the discrete character of the random dot image. If the curvedness of such a surface patch is too low, then this discrete character can affect the 3-D perception. To illustrate this effect, the motion disparity was calculated in terms of pixel displacements per unit of head movement for a shape with $C=1 / \mathrm{m}$ and $S=1.0$ (see Figure 8). As can be seen in this figure, there are only nine levels of motion disparity in such a stimulus. This means, for example, that when one moves from one side of the chinrest to the other side, the dots at the outer corners of the stimulus will move, at most, 9 pixels. Apparently, this information is too coarse for the human visual system to reconstruct a reliable 3-D curvature. This might explain why the $C=1 / \mathrm{m}$ condition deviates strongly from the other values. The results for $C=1 / \mathrm{m}$ are therefore discarded in further analysis. Once more, a two way ANOVA (curvedness $\times$ shape index) was performed on the remaining data of Figure 6. The main effect of curvedness on the JNDs was significant [K.Z., $F(2,45)$ $=7.953, p=.001 ; \mathrm{S} . \mathrm{M} ., F(2,45)=6.405, p=.004$; W.D., $F(2,45)=3.732, p=.032$ ]. The main effect of the shape index was only significant for 1 subject, K.Z. $[F(4,45)=3.415, p=.016]$. There was no significant interaction between shape index and curvedness.

Mean Weber fractions were calculated by linear regression of the data of Figure 6, over the range of $C=5 / \mathrm{m}$ to $20 / \mathrm{m}$. These Weber fractions are plotted in Figure 9. It is clear from Figure 9 that the Weber fractions fluctuate around $15 \%-25 \%$, and that they are roughly the same for all shapes. If it is assumed that sensitivity can be expressed as the reciprocal Weber fraction, then human sensitivity for curvedness does not depend on the shape index.

\section{DISCUSSION}

One of the goals in the experiments was to see whether the discrimination of 3-D quadratic shape is equally good for all quadratic shapes. The results of the present experiments show that this is not the case. Although the differences are not spectacular, there is a significant difference in discriminating between two elliptic-like shapes on the one hand and between two umbilical-like shapes on the other. This important finding warns us not to use just any arbitrary 3-D shape in a 3-D shape perception experiment, especially if we want to generalize results from one 3-D shape to another. Although the present experiment was restricted to quadratic shapes, it is not unlikely that this conclusion also holds for other 3-D shapes. Many experiments concerning the kinetic depth effect or structure from motion have been done with cylinders as the only 3-D shape. Our results show that one must be careful in generalizing from these experiments. It is not clear whether the subjects actually perceived 3-D shape or that they reconstructed 3-D shape from other sources of information. One hypothesis emerges from interviews with the subjects after the shape discrimination sessions. Subjects reported that they often focused their attention on the curvature in the principal directions. Is it therefore possible to do the shape discrimination task by using only the principal curvatures?

If we consider the condition in which the curvedness is fixed, discrimination of 3-D quadratic shape might be performed in two steps: first, the orientation of the principal frame might be extracted. For this operation, the direction of only one principal curvature would do, since the principal directions are orthogonal. (However, subjects were not informed about the orthogonality of the principal frame until all the experimental sessions were finished). The second step could then be the estimation of the magnitude of the principal curvatures. Once this was done, the shape index would be known and the comparison of reference shape index and test shape index could be made. Actually, the comparison of two images could be made without "calculating" the shape index of each image. According to this strategy, observers do not even need an intuitive notion of shape or shape index. However, in the random curvedness situation, the principal curvatures have random magnitudes, so if subjects used the magnitude of the principal curvatures to make 

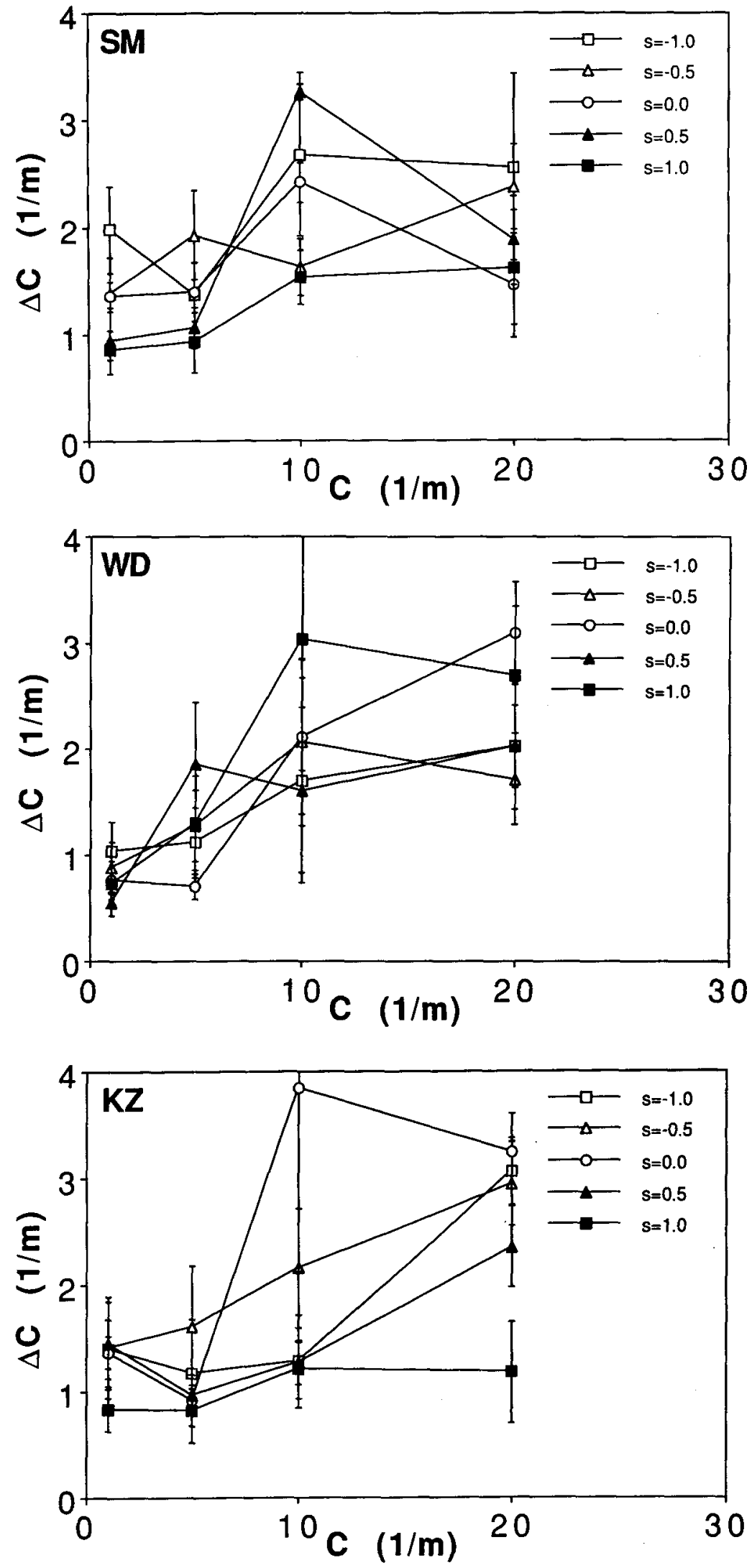

Figure 6. Just noticeable curvedness differences for the 3 subjects S.M., W.D., and K.Z. for the five conditions: $S=-1.0,-0.5,0.0,0.5$, and 1.0. Error bars denote standard errors. 

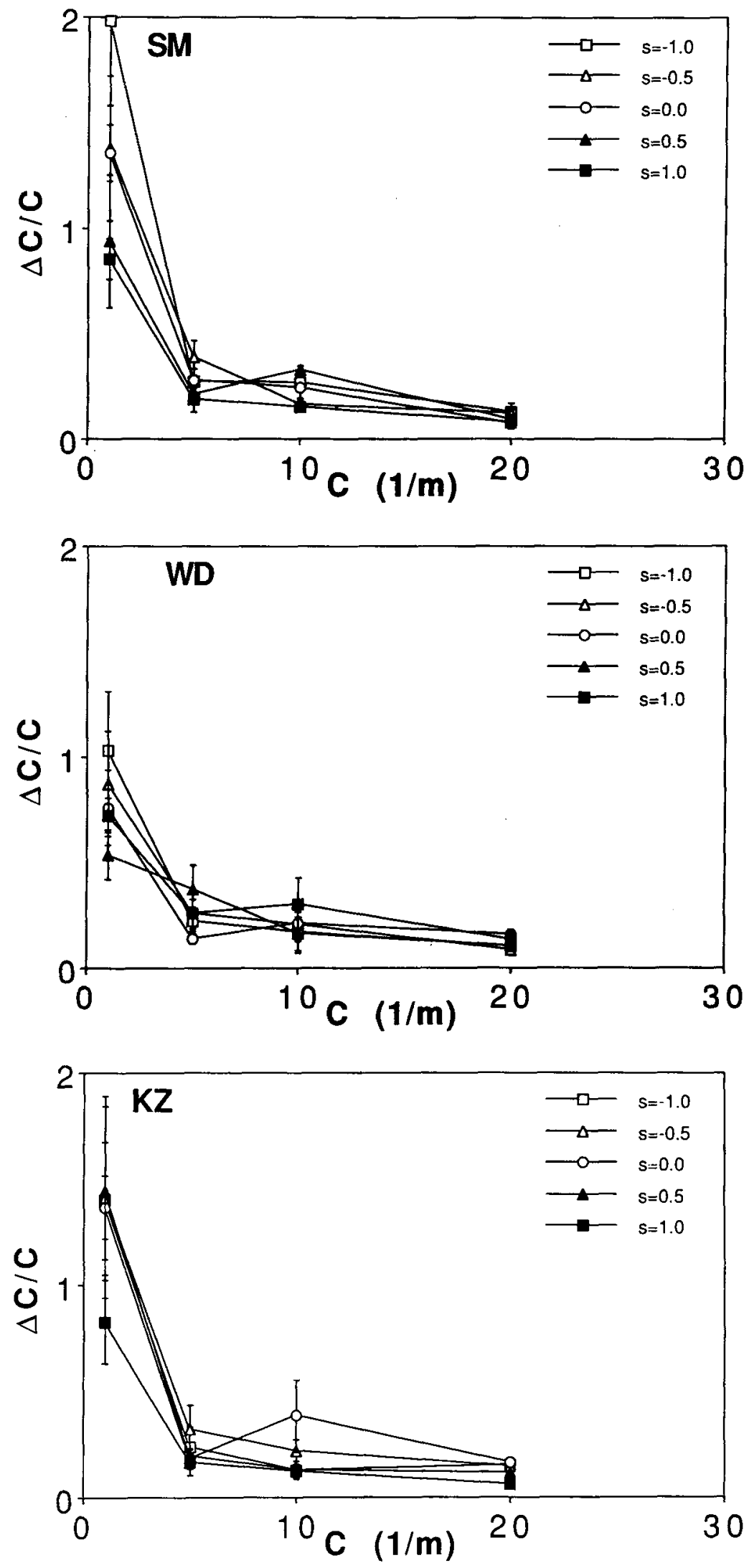

Figure 7. $\triangle C / C$ for the 3 subjects S.M., W.D., and K.Z. for the five conditions: $S=$ $-1.0,-0.5,0.0,0.5$, and 1.0. Error bars denote standard errors. 
the distinction between the two images, this would lead to a chance performance. Since none of the 3 subjects in the present study had a chance performance in the random curvedness situation, we conclude that this strategy was not used in the shape discrimination experiment. In fact, all subjects showed equal performance in the random curvedness condition and in the fixed curvedness condition.

An alternative strategy based on the principal curvatures that would also be compatible with the subjective reports could be that subjects estimate the ratio of the two principal curvatures. If we call this ratio $R$, then the test and reference images have ratios $R_{\text {test }}$ and $R_{\text {ref. }}$. From the definition of curvedness and shape index, it follows that this ratio $R$ does not depend on the curvedness. This strategy would lead to equal performance in both the fixed curvedness and the random curvedness situation, although subjects never explicitly reported that they used this strategy. Computing a ratio of two curvatures in different directions and then comparing it with another ratio at a different time is a rather complex task for the visual system, but we cannot discard this hypothesis on the basis of the results of the present experiment. In fact, there have been reports that the human visual system is quite able to use complex geometric quantities such as lines of minimal curvature in constructing global shape (Braunstein, Hoffman, \& Saidpour, 1989). The present experiment indicates that principal curvatures could be used in the perception of local shape.

The results of Experiment 2 show that increment thresholds for Koenderink's curvedness depend linearly on curvedness (at least within the range of $5 / \mathrm{m}$ to $20 / \mathrm{m}$ ) but not on the shape. This second experiment therefore indicates that not 3-D shape but 3-D curvatures are used in the structure-from-motion process.

For the very special case $S= \pm 0.5$ (parabolic shapes), one of the principal curvatures vanishes. Because even for

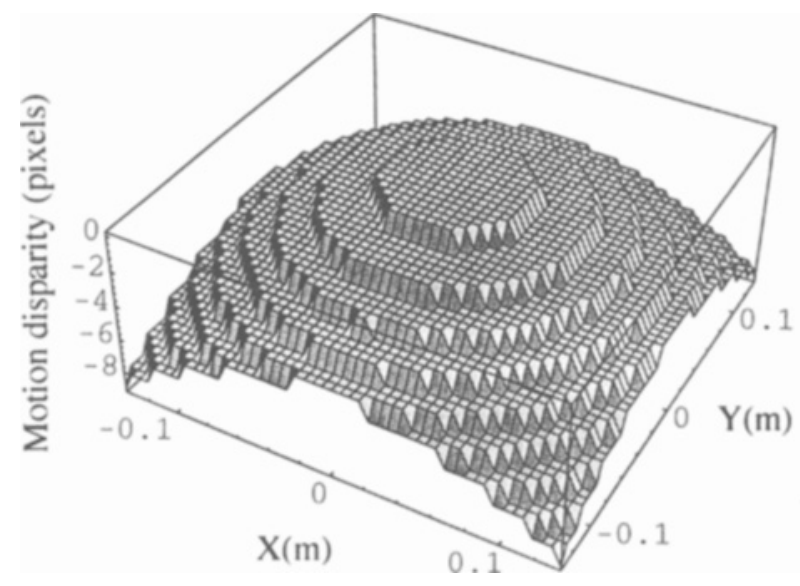

Figure 8. Pixel displacements per unit of head movement for a stimulus with $C=1 / \mathrm{m}$ and $S=1.0$. Clearly visible are the pixel errors that occur in this range of curvedness values. As can be seen, there are only nine levels of motion disparity in this stimulus, ranging from 0 to 9 pixels displacement when the chinrest is moved over the total length of the rail.

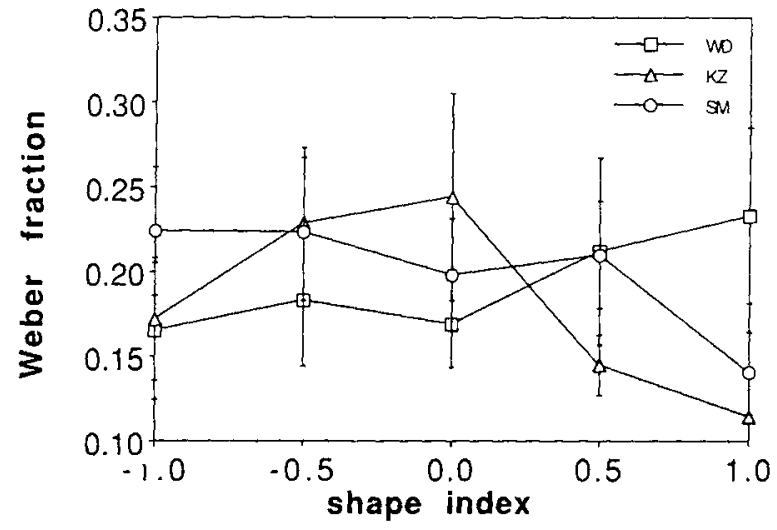

Figure 9. Weber fractions for the 3 subjects S.M., W.D., and K.Z., as a function of shape index. These Weber fractions were found by linear regression analysis of the data of Figure 7 over the range of $C=5 / \mathrm{m}$ to $C=20 / \mathrm{m}$. Error bars denote standard errors.

these shapes a Weber law was found, we may conclude that the discrimination of 3-D curvature (or the curvature of a space curve) obeys Weber's law as well. It is interesting to compare the 3-D curvature case with the 2-D curvature case (the curvature of a curve in the frontoparallel plane). Foster, Simmons, and Cook (1992) and Koenderink and Richards (1988) showed that the discrimination of a 2-D line curvature in the frontoparallel plane followed Weber's law quite well, albeit with smaller fractions (about 10\%; it was $20 \%-30 \%$ in our experiment, or the 3-D situation). It is not unlikely that the perception of 3-D curvature and 2-D curvature have much in common.

Suppose that the discrimination of 3-D quadratic shape is based on the perception and comparison of 3-D curvatures (e.g., the principal curvatures). We might then simulate the shape discrimination results if we assume that subjects use only the smallest (or the largest) of the absolute value of the two principal curvatures in one image. This corresponds to the subjective reports that were mentioned earlier. Figure 10 shows a plot of the principal curvatures as a function of the shape index.

When the absolute values of the two principal curvatures are almost equal $(S \pm 1.0$ and $S=0.0)$, it will be difficult for subjects to judge which one of the two curvatures is the one to use. In this case, the discriminability is mainly determined by the accuracy of finding the smallest (or largest) curvature, and to a lesser degree by the discriminability of 3-D curvature. On the other hand, when one of the two principal curvatures is zero $(S=$ \pm 0.5 ), the difference is maximal, and the subjects should not have much difficulty in finding the smallest (or the largest) curvature. In this case, the discriminability is mainly determined by the discriminability of 3-D curvature. So these two assumptions already predict a $W$-shaped sensitivity curve that resembles the one that was found in Experiment 1.

These simulations therefore show that human observers might perceive 3-D curvatures and reconstruct 3-D shape based on these 3-D curvatures instead of perceiving 3-D 


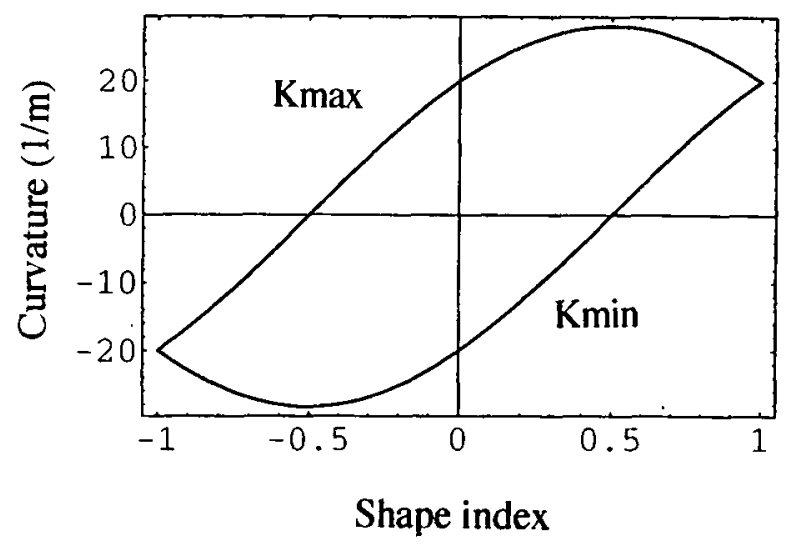

Figure 10. The principal curvatures $K_{\min }$ and $K_{\max }$, as a function of the shape index.

shape at once. Probably, this will not hold for other shapes. Quadratic shapes are obviously simple shapes, in the sense that extraction of the principal frame is likely to be very easy. For 3-D image displays, the fact that curvedness does not influence the JNDs for any quadratic shape means that emphasizing 3-D shape by exaggerating the curvature does not have any effect. Our future research will concentrate on the contribution of active vision to the JNDs measured in this experiment. Is a passive observer (who does not make exploratory head movements) better or worse than an active observer in discriminating two different 3-D quadratic shapes specified by optical motion?

\section{REFERENCES}

Braunstein, M. L., \& Andersen, G. J. (1984). Shape and depth perception from parallel projections of three-dimensional motion. Journal of Experimental Psychology: Human Perception \& Performance, 6, 749.760 .

Braunstein, M. L., Hoffman, D. D., \& Saidpour, A. (1989). Parts of visual objects: An experimental test of the minima rule. Perception, 18, 817-826.

COXETER, H. S. M. (1961). Introduction to geometry. New York: Wiley. DE VRies, S. C., Kappers, A. M. L., \& KoenderinK, J. J. (1993). Shape from stereo: A systematic approach using quadratic surfaces. Perception \& Psychophysics, 53, 71-80.
Dosher, B. A., Landy, M. S., \& SperLING, G. (1989). Ratings of kinetic depth in multidot displays. Joumal of Experimental Psychology: Human Perception \& Performance, 15, 816-825.

Droulez, J., \& Cornilleau-PÉRÈs, V. (1990). Visual perception of surface curvature: The spin variation and its physiological implications. Biological Cybernetics, 62, 211-224.

Foster, D. H., Simmons, D. R., \& Cook, M. J. (1992). The cue for contour-curvature discrimination. Vision Research, 33, 329-341.

Koenderink, J. J. (1990). Solid shape. Cambridge, MA: MIT Press.

KoEnderink, J. J., \& Richards, W. (1988), Two-dimensional curvature operators. Joumal of the Optical Society of America A, 5, 1136-1141.

Landy, M. S., Dosher, B. A., SPerung, G., \& PerkTns, M. E. (1991). The kinetic depth effect and optic flow: II. First- and second-order motion. Vision Research, 5, 859-876.

Norman, J. F., \& LAPPIN, J. S. (1992). The detection of surface curvatures defined by optical motion. Perception \& Psychophysics, 51, 386-396.

Rogers, B. J., \& GrahaM, M. (1979). Motion parallax as an independent cue for depth perception. Perception, 8, 125-134.

Rogers, B. J., \& Graham, M. (1983). Motion parallax and the perception of three-dimensional surfaces. In D. J. Ingle, M. Jeannerod, \& D. N. Lee (Eds.), Brain mechanisms and spatial vision: Proceedings of the NATO Advanced Study Institute on Brain Mechanisms and Spatial Visions (pp. 95-111). Dordrecht: Nijhoff.

Roll, R., Velay, J. L., Roll, J. P. (1991). Eye and neck proprioceptive messages contribute to the spatial coding of retinal input in visually oriented activities. Experimental Brain Research, 85, 423-431.

Saidpour, A., Braunstein, M. L., \& Hoffman, D. D. (1992). Interpolation in structure from motion. Perception \& Psychophysics, 51, 105-117.

Sperling, G., Landy, M. S., \& Dosher, B. A. (1990). How to study the kinetic depth effect experimentally. Joumal of Experimental Psychology: Human Perception \& Performance, 16, 445-450.

Sperling, G., Landy, M. S., Dosher, B. A., \& Perkins, M. E. (1989). Kinetic depth effect and identification of shape. Journal of Experimental Psychology: Human Perception \& Performance, 15, 826-840.

TODD, J. T. (1984). The perception of three-dimensional structure from rigid and nonrigid motion. Perception \& Psychophysics, 36, 97-103.

Todd, J. T., \& Norman, J. F. (1991). The visual perception of smoothly curved surfaces from minimal apparent motion sequences. Perception \& Psychophysics, 50, 509-523.

Treue, S., Husain, M., Andersen, R. A. (1991). Human perception of structure of motion. Vision Research, 1, 59-75.

van Damme, W. J. M., \& VAN de Grind, W. A. (1993). Active vision and the identification of 3D shape. Vision Research, 11, 1581-1587.

WALLACH, H., \& O'CONNELL, D. N. (1953). The kinetic depth effect. Journal of Experimental Psychology, 45, 205-217.

(Manuscript received February 24, 1993; revision accepted for publication August $9,1993$. ) 